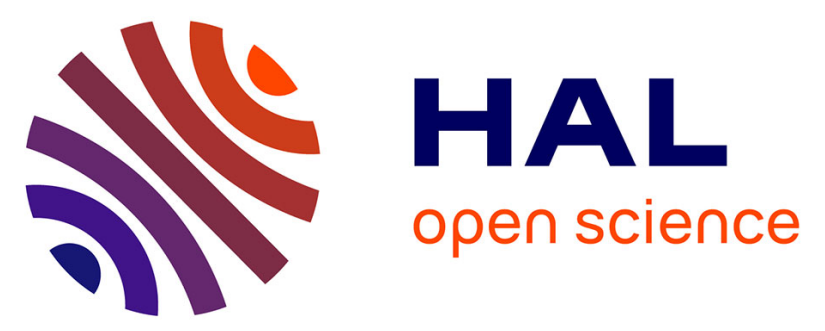

\title{
Les hommes de fer du dieu Ageio: exploitation antique du fer dans les Hautes Baronnies (Hautes-Pyrénées)
} Argitxu Beyrie, Jean-Marc Fabre, Robert Sablayrolles

\section{To cite this version:}

Argitxu Beyrie, Jean-Marc Fabre, Robert Sablayrolles. Les hommes de fer du dieu Ageio: exploitation antique du fer dans les Hautes Baronnies (Hautes-Pyrénées). Gallia - Archéologie de la France antique, 2000, Mines et métallurgies en Gaule, 57, pp.37-52. 10.3406/galia.2000.3208 . hal-01770309

\section{HAL Id: hal-01770309 \\ https://hal.science/hal-01770309}

Submitted on 18 Apr 2018

HAL is a multi-disciplinary open access archive for the deposit and dissemination of scientific research documents, whether they are published or not. The documents may come from teaching and research institutions in France or abroad, or from public or private research centers.
L'archive ouverte pluridisciplinaire HAL, est destinée au dépôt et à la diffusion de documents scientifiques de niveau recherche, publiés ou non, émanant des établissements d'enseignement et de recherche français ou étrangers, des laboratoires publics ou privés.

\section{(1) (1) $\$$}

Distributed under a Creative Commons Attribution - NonCommercial - NoDerivatives 44.0 


\title{
Les hommes de fer du dieu Ageio. Exploitation antique du fer dans les Hautes Baronnies (Hautes-Pyrénées)
}

In: Gallia. Tome 57, 2000. pp. 37-52.

\begin{abstract}
Due to a four years archaeological survey with ponctual excavations, it has been possible to draw up a map of the mining and metallurgical remains in the Hautes Baronnies (Hautes-Pyrénées) and to make clear the existence of an antic iron mining and metallurgy. Extraction and production are now attested during Roman times. The map gives a picture of the mining and metallurgical organization. An analysis of the existing epigraphical evidence proves that this region was actually the pagus ferrariensis of the Asque's inscription and opens up prospects on the workers' status.
\end{abstract}

\section{Résumé}

Quatre années de prospections et de sondages d'évaluation archéologique ont permis de dresser une carte des vestiges miniers et métallurgiques des Hautes Baronnies (Hautes-Pyrénées) et de démontrer l'existence d'une phase antique de l'exploitation du fer. L'analyse de la carte offre une image de l'organisation de l'exploitation. L'examen des données épigraphiques existantes prouve que ce secteur est bien le pagus ferrariensis de l'inscription d'Asque et ouvre des perspectives sur la condition des exploitants.

Citer ce document / Cite this document :

Beyrie Agitxu, Fabre Jean-Marc, Sablayrolles Robert. Les hommes de fer du dieu Ageio. Exploitation antique du fer dans les Hautes Baronnies (Hautes-Pyrénées). In: Gallia. Tome 57, 2000. pp. 37-52.

doi : $10.3406 /$ galia.2000.3208

http://www.persee.fr/web/revues/home/prescript/article/galia_0016-4119_2000_num_57_1_3208 


\title{
LES HOMMES DE FER DU DIEU AGEIO
}

\author{
Exploitation antique du fer dans les Hautes Baronnies \\ (Hautes-Pyrénées)
}

\author{
Agitxu Beyrie, Jean-Marc Fabre et Robert SABlayrol.tes
}

\begin{abstract}
Mots-clés. Pyrénées, mélallurgie, mine, fer, Antiquité, épigraphie.
Key-words. Pyrénées, metallurgy, mining, iron, Anliquily, eprigraphy.

Résumé. Quatre années de prospeciions el de sondages d'évaluation archéologique ont permis de dresser une carle des vestiges miniers et mélallurgiques des Hautes Baronnies (Hautes-Pyrénées) el de démontrer l'existence d'une phase antique de l'exploitation du fer. L'analyse de la carte offre une image de l'organisation de l'exploitation. L'examen des données épigraphiques existantes prouve que ce secteur est bien le pagus ferrariensis de l'inscription d'Asque et ouvre des perspectives sur la condition des exploilants.
\end{abstract}

\begin{abstract}
Due to a four years archaeological survey with ponctual excavations, it has been possible to draw up a map of the mining and metallurgical remains in the Hautes Baronnies (Hautes-Pyrénées) and to make clear the existence of an antic iron mining and metallurg): Extraction and production are now allested during Roman times. The map gives a picture of the mining and metallurgical mganization. An analysis of the existing epigraphical evidence proves that this region was actually the pagus ferrariensis of the Asque's inscription. and opens up prospects on the workers' status.
\end{abstract}

L'histoire s'écrit souvent à coups de formules toutes faites et de légendes. Diodore de Sicile évoquait ainsi les rivières d'argent pur que l'écobuage de bergers maladroits avait fait couler des flancs des Pyrénées (Diodore, Bibliothèque historique, 5, 33), la geste épique des comtes de Foix compare leur rude puissance et leur éclat politique au fer du Vicdessos et à l'or de l'Ariège et, de nos jours, les masses métalliques du Canigou passent pour dérégler les appareils de navigation aérienne, entraînant plusieurs catastrophes dans le massif. Ainsi se dessine l'image d'Épinal de Pyrénées généreuses, éternellement pourvoyeuses d'or, d'argent, de fer, de cuivre et de plomb pour les aventuriers de toutes les époques. On ne prête qu'aux riches, et la tradition minière et métallurgique du monde pyrénéen, certes largement attestée du Bronze ancien jusqu'à l'époque contemporaine, a parfois entraîné en la matière des excès condamnables sur le plan de la méthode d'analyse. Il ne suffit pas, en effet, d'un faisceau d'indices épars (données géologiques, quelques repères chronologiques d'époques diverses, une ou deux remarques générales tirées de textes antiques cent fois utilisés) pour reconstruire, dans un secteur déterminé des Pyrénées, le schéma d'une activité minière ou métallurgique d'époque antique. Pour étayer semblables hypothèses, il faut l'appui d'une argumentation archéologique solide, issue de prospections et de fouilles suffisamment poussées et étendues pour donner quelque crédit aux conclusions.

Telle est l'expérience qui a été entreprise dans les Hautes Baronnies, district montagneux des HautesPyrénées, qui abrite les sources de l'Arros et son bassin supérieur. L'exploitation du fer s'y pratiquait encore au début du XX $\mathrm{XX}^{\mathrm{e}} \mathrm{s}$., où fonctionnaient, dans la vallée d'Aure, les forges de Rebouc (commune de Hèches), qui utili- 
saient un minerai issu des vallées voisines. Auparavant, le baron de Dietrich, en 1786, décrivait sommairement un puits de 400 pieds de profondeur, qui alimentait une forge à Hèches même, puits qu'il est tentant d'identifier avec une des minières de la Crête de Sarramer (fig. 16), qui sépare le vallon du Bouchidet, à l'est, de celui de l'Artiguette affluent de l'Arros, à l'ouest ${ }^{47}$. C'est probablement cette même crête qui fit l'objet, en 1646, d'un litige de limite entre le seigneur de Bégole, propriétaire d'une mine vers Esparros, et le seigneur d'Ossun, propriétaire sur le territoire de Hèches ${ }^{48}$. Enfin, la redécouverte récente, dans l'église d'Asque, de l'autel votif élevé par des pagani ferrarienses à la divinité topique Ageio (fig. 18) paraissait porter témoignage d'une phase antique de l'exploitation (CIL XIII, 384) ${ }^{49}$. Ainsi se trouvait réuni un faisceau d'indices attestant l'exploitation du fer à diverses époques, mais la source épigraphique, à elle seule, ne suffisait pas, malgré le terme explicite de pagani ferrarienses, à attribuer à l'Antiquité romaine tout ou partie des vestiges miniers et métallurgiques dispersés sur les crêtes et dans les vallons du haut bassin de l'Arros et de celui du Bouchidet ${ }^{50}$.

C'est à vérifier archéologiquement cette hypothèse d'une phase antique de l'exploitation du fer dans les Hautes Baronnies que furent consacrées les quatre

47. Baron de Dietrich, Description des gîtes de minerai, des forges et des salines des Pyrénées, Paris et Strasbourg, 1786, p. 324-325.

48. P. Claracq, Présentation de la vallée et ressources du sous-sol, in : $L a$ vallée de l'Arros depuis la Préhistoire, occupation du sol et vie quotidienne à travers l'archéologie et l'histoire, Tarbes, Association Guillaume-Mauran, 1995, p. 3-6.

49. Cf. note 48 : P. Claracq, op. cit., 1995, p. 5 ; R. Sablayrolles, L.es dieux pyrénéens: de farouches montagnards ou d'accueillants indigènes?, in: Divinidades indigenas e interpretatio romana, II Coloquio internacional de Epigrafia, Sintra (Portugal), 16-18 mars 1995, à paraître; R. Sablayrolles, Le pagus dans le cadre pyrènéen, in: L'organisation des espaces antiques : entre nature et histoire, Actes du colloque international de Pau, 21-22 mars 1997, Pau, 2000, p. 111-132.

50. L'inscription, copiée au XV' $\mathbf{s}$., était depuis en remploi dans un mur de la sacristie de l'église et dissimulée par un enduit. Sa provenance comme son authenticité avaient fait l'objet d'hypothèses diverses (voir R. Lizop, Histoire de deux cités gallo-romaines. Les Convenae et les Consoranni, Toulouse, 1931, p. 67 et 231 ; J.J. Martin, Autels votifs de Bagnères-de-Bigorre, Bulletin de la société Ramom, 1970, p. 30-33; cf. note $49: \mathrm{R}$. Sablayrolles, op. cit., 2000). Elle ne figure pas dans le recueil épigraphique de J. Sacaze (Inscriptions antiques des Pyrénées, Toulouse, 1892) ni dans celui de P. Wuilleumier (Inscriptions latines des Trois Gaules, XVIr suppl. à Gallia, Paris, 1963), les deux auteurs ayant sans doute jugé suspecte la copie effectuée au XVI's. par l'érudit Oihenart. années de prospections effectuées de 1995 à 1998 par l'équipe de recherche de l'université de ToulouseLe Mirail, encadrée par A. Beyrie, J.-M. Fabre et R. Sablayrolles ${ }^{51}$. Une première année d'investigation très générale (dépouillement bibliographique, enquête orale, parcours sommaire du terrain) fut suivie de prospections systématiques des différentes vallées, destinées à établir une carte précise des vestiges miniers et métallurgiques. Ces prospections furent complétées de sondages archéologiques sur diverses structures (crassiers de scories notamment) afin d'établir une chronologie assurée des vestiges les plus significatifs. Les résultats, dont l'inventaire est donné sous forme de catalogue, permettent une première synthèse qu'il faudra compléter, quelques secteurs marginaux requérant des compléments d'investigation et l'exploitation des données en termes d'analyse technique n'ayant été que partiellement réalisée. Avant cet inventaire analytique et l'exposé des premières conclusions qu'il met en évidence, il faut tracer le cadre géographique, topographique et géologique de cette étude.

\section{LE MILIEU : DES VALLÉES CALCAIRES AU RELIEF ESCARPÉ ET ABONDAMMENT BOISÉ}

Au centre de la chaîne des Pyrénées, la région des Baronnies s'étend d'est en ouest sur $15 \mathrm{~km}$, de la vallée de l'Adour (Bagnères-de-Bigorre) à la vallée de la Neste d'Aure (Sarrancolin). Elle se situe sur la ligne de partage des eaux qui alimentent le bassin de l'Adour et celui de la Garonne. Du nord au sud, sur $8 \mathrm{~km}$, ce secteur de moyenne montagne au relief très cloisonné est enserré par le plateau de Lannemezan $(600 \mathrm{~m})$ et le massif du Bassia $(1921 \mathrm{~m})$. Soumis à une forte influence océanique, le climat montagnard se caractérise par de fréquentes précipitations. Le relief relativement doux de la partie septentrionale des Baronnies a suscité la concentration de l'habitat en fond de vallée, le développement de l'élevage bovin et, dans une moindre mesure, la pratique d'une polyculture traditionnelle

5l. Ces recherches se sont inscrites dans le cadre de travaux universitaires : A. Beyrie, A. Jacquet, Prospection-inventaire des communes de Nistos, Hèches, Fsparros, Asque, mém. de Maîtrise (sous la dir. de R. Sablayrolles), univ. de Toulouse II, 1995, stages de prospection. 


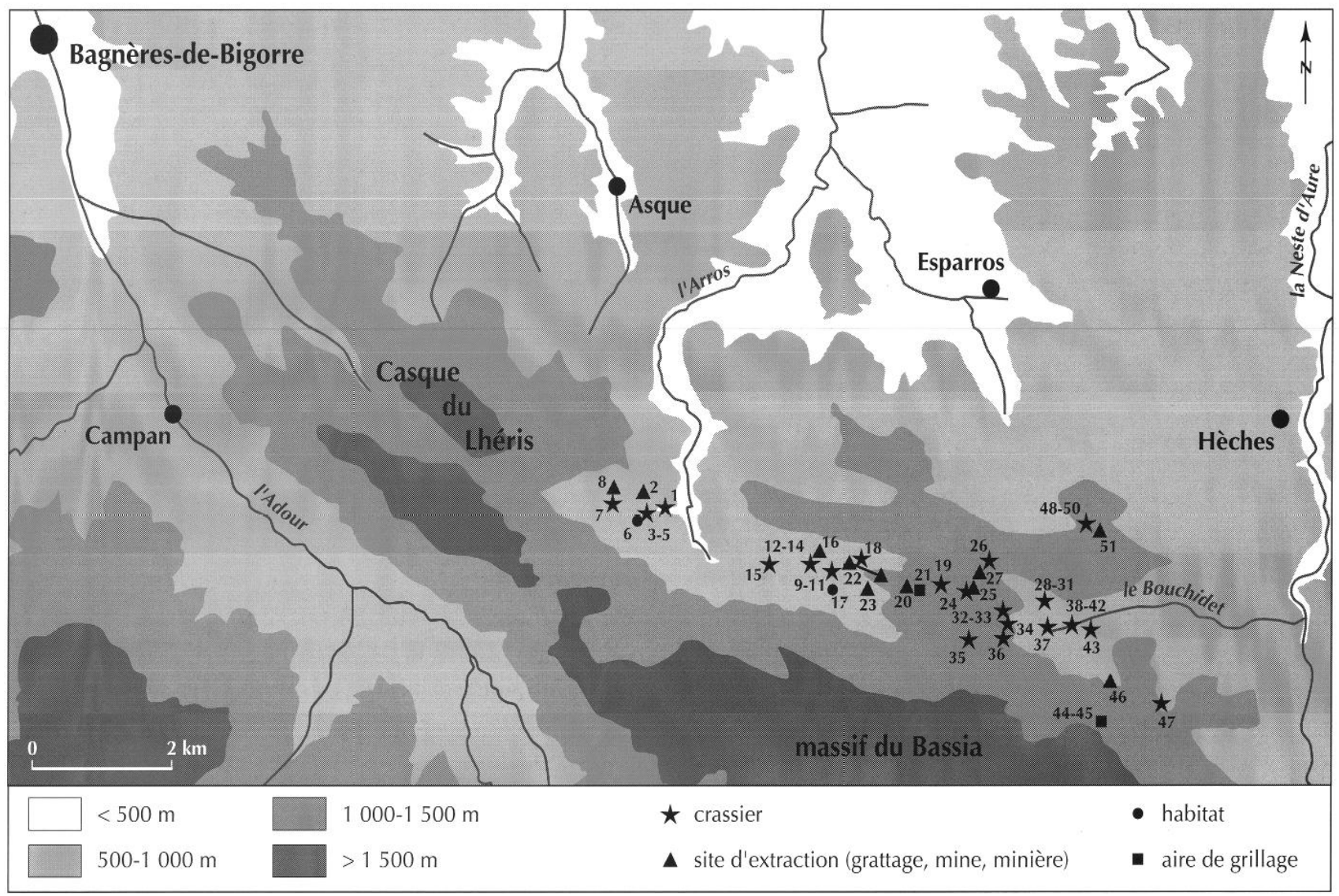

Fig. 10 - Carte générale des Hautes Baronnies avec localisation des sites.

en zone de piémont. Au sud, en revanche, dans la partie la plus montagneuse, au-dessous des estives $(1500 \mathrm{~m})$, se développe un important couvert forestier, nettement dominé par le hêtre. Les petites vallées, ou coumes, sont très encaissées, humides, et envahies par la végétation. Les pentes escarpées, où se détachent par endroits des falaises calcaires de plusieurs dizaines de mètres de haut, sont impropres à toute forme d'élevage et de culture et donnent à l'espace un aspect sauvage et inhospitalier.

Les traces d'exploitations minières et métallurgiques repérées se concentrent dans la partie méridionale des Baronnies, sur les communes d'Asque, Esparros et Hèches (fig. 10). Au centre de cette zone, le col des Estrets matérialise la ligne de partage des eaux : à l'ouest de ce point, le flux hydrographique des vallées et des coumes débouche sur Asque et alimente l'Adour, tandis que le réseau oriental se dirige vers Hèches et la Neste pour rejoindre la Garonne. Ainsi, deux ensembles topographiques se caractérisent-ils.
- Au centre du secteur occidental, l'Arros, de direction sud-nord est la vallée la plus importante (fig. 10 et 11). Son cours d'eau voit le jour à l'Oueil de l'Arros (586 m) et s'écoule vers le nord. De part et d'autre, deux coumes drainent des eaux qui alimentent ce dernier dans son tronçon méridional : à l'ouest, au pied de la montagne de Billexe, depuis le col du Lhéris (1 $383 \mathrm{~m}$ ) jusqu'à l'Arros, une vallée très étroite, humide, et d'accès difficile, la Coume du Lhéris, dans laquelle aboutissent trois petites coumes sèches de direction nord-sud (fig. 11) ; à l'est, au sud de la Crête de l'Escala (1 179 m), la Coume et le ruisseau de l'Artiguette, ralliés au sud-ouest de la Crête de Sarramer (1 $179 \mathrm{~m}$ ) par le ravin d'Inher à $797 \mathrm{~m}$ d'altitude (fig. 12).

- Dans l'ensemble oriental, d'ouest en est, les Coumes des Estrets et de Castillon ainsi que les petites coumes affluentes débouchent à $851 \mathrm{~m}$ d'altitude sur la vallée plus ouverte et moins abrupte du Bouchidet (fig. 13), qui rejoint la Neste à hauteur du hameau de Rebouc (Hèches) $(600 \mathrm{~m})$. 


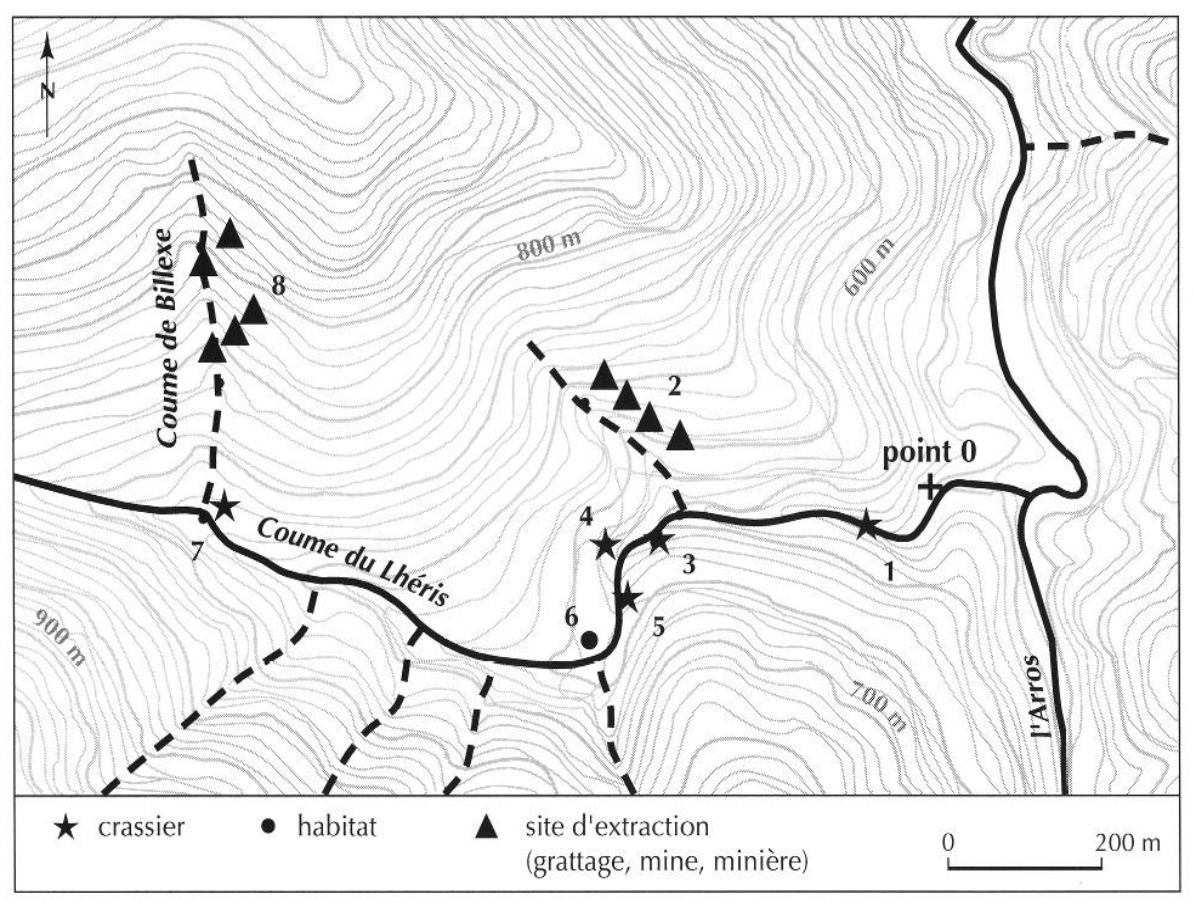

Fig. 11 - La Coume du Lhéris (Hautes Baronnies). Altitudes : équidistance des courbes $=10 \mathrm{~m}$.
L'orogenèse pyrénéenne a, par le biais de la faille nord-pyrénéenne, scindé la région en deux grandes unités géographiques et géologiques bien distinctes.

$\Lambda \mathrm{u}$ sud, la zone axiale, ou haute chaine, se caractérise par des terrains paléozoïques. Ainsi, immédiatement au sud du massif du Bassia, affleure la série des marbres qui ont fait la renommée de la région: Sarrancolin dans la vallée de la Neste et Campan dans la vallée de l'Adour. Au nord, la zone nord-pyrénéenne est constituée de terrains secondaires, essentiellement des formations carbonatées. L'ensemble du secteur étudié se trouve dans cette dernière. Tous les indices minéralisés relevés à l'ouest de l'interfluve (Estrets/Oueil Luzent), notamment dans la vallée de l'Artiguette, sont encaissés dans des dolomies (Infralias) intercalées entre des calcaires de l'Hettangien supérieur et des formations triasiques (pélites ou cargneules) ${ }^{52}$. L'ensemble constitue un anticlinal d'axe nord $115^{\circ}$ est, déversé vers le sud et dont les deux flancs présentent un pendage de $75^{\circ}$. Le minerai de fer, l'anké-

52. J.-L. Raymond, Description et conditions de formation des gîtes ankéritiques pyrénéens: importance des actions thermogravitationnelles, Thèse de 3 cycle, univ. de Toulouse III, 1974, 93 p. ; E.-J. Debroas, Extension et contexte géologique des anciennes carrières de minerais de fer d'Esparros (Hautes-Pyrénées), in: La vallée de l'Arros depuis la Préhistoire, occupation du sol et vie quotidienne à travers l'archéologie et l'histoire, Tarbes, Association Guillaume-Mauran, 1995, p. 7-8. rite, représente 15 à $30 \%$ de la roche encaissante. Deux principaux types de minéralisation ont pu être identifiés : dans les dolomies massives, mais veinées, les fractures sont colmatées par l'ankérite et la teneur est alors de $15 \%$; ou bien celle-ci est interstratifiée et constitue des niveaux millimétriques à centimétriques de couleur rouille : ces couches ont une faible puissance, variant de 0,5 à $1 \mathrm{~m}$, mais la teneur en ankérite atteint $30 \%$. Enfin, plus rarement, s'est constituée une brèche à éléments dolomito-ankéritiques de 2 à $8 \mathrm{~cm}$, cimentés par de l'ankérite.

À l'est, dans la vallée du Bouchidet, la minéralisation est quelque peu différente puisqu'il s'agit d'oligiste, qui constitue le ciment d'une brèche à éléments divers. Cette brèche s'intercale dans des dolomies et des calcaires qui forment une couche de même position stratigraphique que les dolomies ferrifères de la partie occidentale. Cependant, l'ensemble forme ici un monoclinal de direction nord $80^{\circ}$ est et d'un pendage de $80^{\circ}$ nord.

La faible puissance des couches a, semble-t-il, conditionné l'implantation de multiples exploitations dispersées sur les affleurements qui nécessitaient un travail de déblaiement préalable peu important. De plus, selon F. Tollon, il est vraisemblable que les premiers mineurs aient essentiellement exploité les zones superficielles (chapeaux de fer), enrichies en oxydes et hydroxydes de 
fer, renfermant donc des teneurs en fer plus élevées que le minerai originel (ankérite), et dont le traitement par grillage n'était pas nécessaire.

\section{UNE OCCUPATION DU SOL MARQUÉE PAR L'ACTIVITÉ MINIÈRE ET MÉTALLURGIQUE : INVENTAIRE DES SITES}

\section{LE SECTEUR OCCIDENTAL (fig. 10 à 12)}

\section{Coume DU LHÉRIS (fig. 10 et 11)}

Étant donné les difficultés de repérage dans cette vallée très étroite, toutes les localisations ont été effectuées au topofil, à partir du confluent du Lhéris et de l'Arros et d'une cote d'altitude prise à l'altimètre.

Les numéros qui suivent correspondent à ceux des figures 10 à 13 .

1. En remontant dans la vallée, à $350 \mathrm{~m}$ du confluent et $565 \mathrm{~m}$ d'altitude : crassier de $10 \mathrm{~m} \times 5 \mathrm{~m}$, sur la rive gauche du ruisseau.

2. À $500 \mathrm{~m}$ du confluent, dans la petite coume orientée nord-sud, affluent rive gauche du Lhéris, entre 690 et $600 \mathrm{~m}$ d'altitude : trois zones de grattages et sous-cavages, sur $80 \mathrm{~m}$ de longueur, ainsi qu'un dépilage souterrain. De la céramique moderne est présente à l'intérieur des excavations.

3. À $550 \mathrm{~m}$ du confluent et à $605 \mathrm{~m}$ d'altitude : épandage de scories sur une petite butte de quelques mètres, sur la rive droite.

4. À $700 \mathrm{~m}$ du confluent et à $625 \mathrm{~m}$ d'altitude : petit crassier de $1,5 \mathrm{~m} \times 2 \mathrm{~m}$, pour une épaisseur de 0,3 à $0,4 \mathrm{~m}$.

5. À $770 \mathrm{~m}$ du confluent et à $625 \mathrm{~m}$ d'altitude : crassier de $6 \mathrm{~m} \times 2,5 \mathrm{~m}$, pour une épaisseur de $0,5 \mathrm{~m}$, situé sur la rive droite.

6. À $910 \mathrm{~m}$ du confluent et à $640 \mathrm{~m}$ d'altitude, sur un vaste replat de $70 \mathrm{~m} \times 50 \mathrm{~m}$ : habitat caractérisé par les vestiges de plusieurs aménagements en pierres sèches et des concentrations de mobilier sur les petites terrasses en bordure du ruisseau. Sur la rive gauche, contre une paroi rocheuse, une importante quantité de mobilier céramique médiéval et moderne a été recueillie.

7. À $1370 \mathrm{~m}$ du confluent et à $725 \mathrm{~m}$ d'altitude, au débouché de la Coume de Billexe dans la Coume du Lhéris : crassier dont les dimensions n'ont pu être relevées.

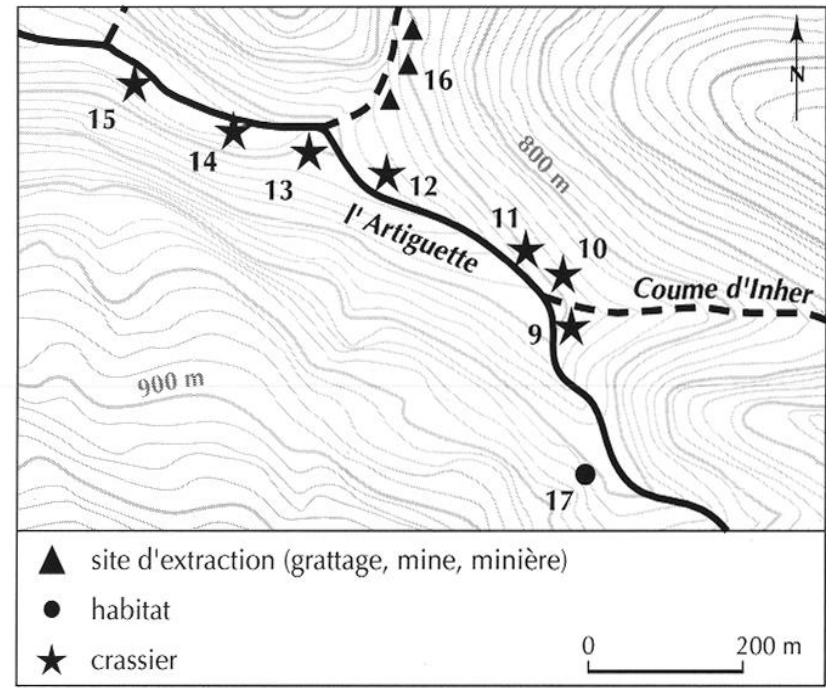

Fig. 12 - La vallée de l'Artiguette (Hautes Baronnies). Altitudes : équidistance des courbes $=10 \mathrm{~m}$.

Coume de Billexe (fig. 10 et 11)

8. De 210 à $340 \mathrm{~m}$ depuis le confluent de la Coume de Billexe avec celle du Lhéris, et entre 785 et $850 \mathrm{~m}$ d'altitude: ensemble de travaux miniers comprenant quatre sous-cavages (longueur maximale $=10 \mathrm{~m}$ ) et d'importants grattages. Une dizaine de fragments de céramique médiévale et moderne ont été découverts dans ces travaux. Le lot de mobilier céramique recueilli dans les excavations minières $n^{\circ} 8$ forme un ensemble cohérent avec tout le matériel du site $n^{\circ} 6$. Cet habitat, peut-être saisonnier, doit très vraisemblablement être mis en relation avec l'extraction minière et les activités métallurgiques de la vallée.

\section{Coume De L'ArTiguette (fig. 10 e. 12)}

9. Au confluent de la Coume d'Inher et de la vallée de l'Artiguette, rive droite de l'Artiguette et gauche de la Coume d'Inher: crassier de $30 \mathrm{~m}$ x $20 \mathrm{~m}$. L'abondant mobilier céramique mis au jour lors d'un sondage date $d u I^{\text {er }}$ au $V^{e} s$. de notre ère.

10. Au confluent de la Coume d'Inher et de la vallée de l'Artiguette, rive droite de l'Artiguette et de la Coume d'Inher : crassier de $15 \mathrm{~m}$ x $12 \mathrm{~m}$.

11. Près du confluent de la Coume d'Inher et de la vallée de l'Artiguette, $50 \mathrm{~m}$ en aval des crassiers $\mathrm{n}^{\text {os }} 9$ et 10 : crassier de $28 \mathrm{~m} \mathrm{x} 15 \mathrm{~m}$. 


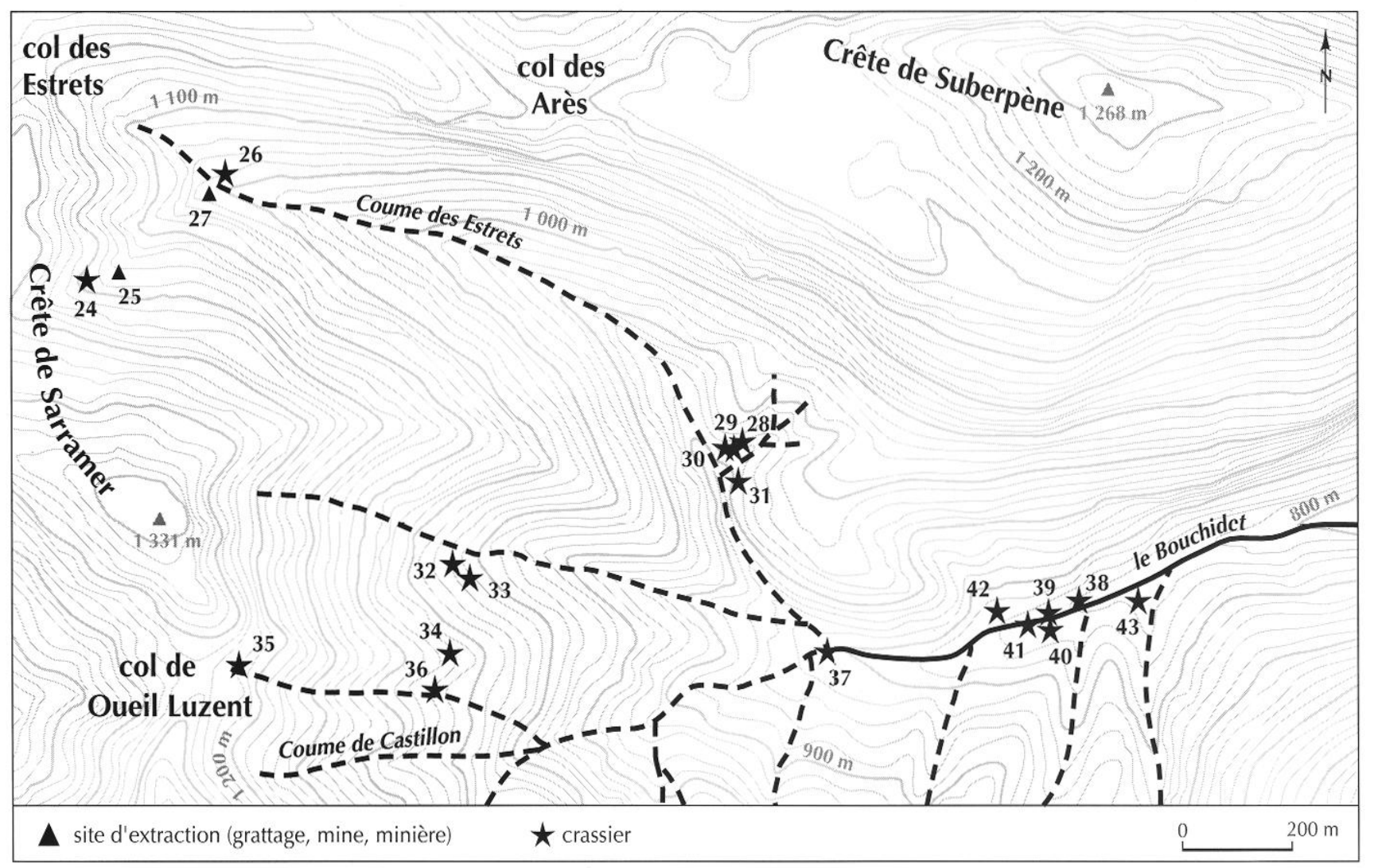

Fig. 13 - La vallée du Bouchidet (Hautes Baronnies). Altitudes : équidistance des courbes $=10 \mathrm{~m}$.

12. À $250 \mathrm{~m}$ en aval des crassiers $\mathrm{n}^{\circ \times} 9-11$, en bordure du ruisseau, rive droite : petit crassier dont le diamètre est inférieur à $10 \mathrm{~m}$.

13. À $120 \mathrm{~m}$ en aval du crassier $\mathrm{n}^{\circ} 12$, au confluent d'une coume nord-sud et de la vallée de l'Artiguette, rive droite de l'Artiguette: crassier de petites dimensions.

14. Sur la rive gauche de l'Artiguette, à $100 \mathrm{~m}$ en aval du crassier $n^{\circ} 13$ : crassier de petites dimensions.

15. Au bord de l'Artiguette, à $700 \mathrm{~m}$ en aval du crassier $\mathrm{n}^{\circ} 14$ : crassier de petites dimensions.

16. Sur le versant oriental de la coume orientée nordsud, au nord des crassiers n ${ }^{\text {ss }} 12$ et $13:$ nombreuses traces de grattage.

17. Sur la rive gauche de l'Artiguette, quelques mètres en amont du crassier $\mathrm{n}^{\circ} 9$ : abri sous-roche de $10 \mathrm{~m} \times 10 \mathrm{~m}$. Des tessons de céramique, découverts dans l'abri et dans ses abords immédiats, témoignent d'une occupation médiévale et moderne du site. Cet habitat temporaire est peut-être lié à certaines activités minières et métallurgiques de la vallée.

\section{Illustration non autorisée à la diffusion}

Fig. 14 - Vue générale des minières de la Crête de Sarramer (IIaules Baronnies) (cliché R. Sablayrolles).

COUME DES ARÈs (fig. 10)

18. Sur le versant méridional de la Coume des Arès, près de la crête séparant la Coume des Arès de la Coume d'Inher: crassier d'une quinzaine de mètres de 
Fig. 15 - Relevé lopographique des minières de la Crête de Sarramer (Hautes Baronnies) (M. Fincker, J.-M. Labarthe, IRAA-CNRS, Pau).

diamètre. L'ensemble du mobilier mis au jour lors du sondage du crassier présente les caractéristiques de productions du Irr s. de notre ère.

\section{Crête de SARRAmer (fig. 10)}

19. Au col de Sarramer : un ferrier d'une quinzaine de mètres de diamètre sur 0,90-1,30 m d'épaisseur. L'ensemble du mobilier céramique recueilli lors du sondage du crassier, notamment de la céramique à paroi fine métallescente, constitue un lot relativement cohérent des deux premiers siècles de notre ère.

20. Sur la Crête de Sarramer: une quinzaine de minières alignées selon l'axe du filon (fig. 14 et 15). Les excavations les plus imposantes se situent dans la moitié orientale de la crête. Elles ont un diamètre de 45-50 m pour une profondeur de $15-20 \mathrm{~m}$ (fig. 16).

21. À l'extrémité sud-est de la Crête de Sarramer, en bordure de la première minière : une structure bâtie en pierres sèches, de forme circulaire, a été fouillée. Il s'agit d'un four de grillage, comme l'indique le seul niveau archéologique relevé dans la stratigraphie, constitué d'une couche de minerai de fer et d'une couche de charbon de bois. La structure, relativement bien conservee, était recouverte par une simple couche d'humus. Il semble qu'elle soit d'époque récente.

22. Sur la crête entre la Coume des Arès et celle d'Inher: tranchées d'exploitation minière, de 10-15 m 


\section{Illustration non autorisée à la diffusion}

Fig. 16 - Vue d'une minière de la Crête de Sarramer (Hautes Baronnies) (cliché R. Sablayrolles).

de largeur, orientées est-ouest, le long d'une falaise calcaire. Ces tranchées se développent sur $1000 \mathrm{~m}$ de long environ. C'est donc l'ensemble du filon qui a été exploité depuis le haut de Sarramer jusqu'à l'extrémité occidentale de la crête entre les Coumes d'Inher et des Arès.

À l'ensemble de Sarramer doit être joint le petit sanctuaire antique fouillé par J.-L. Schenck et son équipe en 1996 (fig. 17). Il se présentait sous la forme d'une terrasse très sommairement aménagée, peut-être munie d'une couverture en bois. Les offrandes qui subsistaient in silu comprenaient plusieurs monnaies, datées du II ${ }^{\mathrm{e}}$ au $V^{\prime}$ s. de notre ère, et des fragments d'autels votifs dont un portait encore, très érodée, une dédicace épigraphique à la divinité Ageio. Quelques vestiges d'opération métallurgique (fragments de fer, scories) ont également été récupérés dans le sol du sanctuaire ${ }^{53}$.

\section{Coume D'INHER (fig. 10)}

23. Sur le versant nord de la Coume d'Inher : trois entrées de mine. Ces galeries permettaient probablement d'accéder aux dépilages de Sarramer.

53. Nous remercions vivement J.-I. Schenck et son équipe de nous avoir transmis ces renseignements et de nous avoir autorisés à les utiliscr. Une publication détaillée de la fouille du sanctuaire doit paraitre.

\section{Illustration non autorisée à la diffusion}

Fig. 17 - Vue générale du col de Sarramer et de la Coume des Arès (Hautes Baronnies) : au premier plan, minières de Sarramer; à l'arrière plan, crassier du col et sanctuaire (cliché R. Sablayrolles).

LE SECTEUR ORIENTAL (fig. 10 et 13)

Coume Des Estrets (fig. 10 et 13)

24 et 25. Au pied de la Crête de Sarramer, en haut d'une petite coume aflluent rive gauche de la Coume des Estrets : crassier et traces d'exploitation du fer.

26 et 27. Au confluent d'une petite coume affluent rive gauche de la Coume des Estrets : crassier de $10 \mathrm{~m}$ de diamètre et départ de galerie de mine.

28, 29, 30 et 31. Dans la dernière coume affluent, sur le versant nord, de la Coume des Estrets : quatre petits crassiers (moins de $50 \mathrm{~m}^{2}$ de superficie). Le mobilier céramique recueilli dans les sondages des crassiers $n^{\text {os }} 28$ et 31 peut être daté de l'Antiquité.

\section{LE VALLON ENTRE LES COUMES DES ESTRETS et de Castillon (fig. 10 et 13)}

32. Au milieu du vallon, sur le versant méridional : crassier de $10 \mathrm{~m} \times 12 \mathrm{~m}$ sur $0,80 \mathrm{~m}$ d'épaisseur. Le mobilier ramassé en surface présente des caractères anciens, mais ne peut être daté avec précision.

33. À $30 \mathrm{~m}$ en aval du $\mathrm{n}^{\circ} 32$ : quelques scories en surface, épandage de scories provenant du crassier $\mathrm{n}^{\circ} 34$ ?

34. Au sommet du versant entre le vallon et la Coume de Castillon, à mi-pente entre la Crête de Sarramer et la 
vallée du Bouchidet : crassier de $20 \mathrm{~m}$ x $10 \mathrm{~m}$ pour une épaisseur de $1 \mathrm{~m}$.

Coume de Castillon (fig. 10 et 13)

35. Sur le col de Oueil Luzent : crassier détruit par l'aménagement d'une piste forestière.

36. Dans le dernier affluent de la rive gauche de la Coume de Castillon : petit crassier de $10 \mathrm{~m} \times 5 \mathrm{~m}$.

\section{La valú́e DU Bouchmet (fig. 10 et 13)}

37. Au bord du parking aménagé sur la piste forestière: crassier en grande partie détruit par l'aménagement du parking ; un sondage, effectué en 1998, n'a pas permis de dater précisément le site ; on peut simplement noter l'absence de céramique moderne. Une datation par radiocarbone, sur des charbons de bois prélevés, est en cours.

Les sites suivants sont repérés par rapport à la cabane forestière du Bouchidet.

38. Contre la cabane, à l'est : crassier de $35 \mathrm{~m}$ x $15 \mathrm{~m}$ pour une épaisseur de $0,8 \mathrm{~m}$.

39. Sous la cabane : crassier de $10 \mathrm{~m}$ x $5 \mathrm{~m}$ sur $0,5 \mathrm{~m}$ d'épaisseur.

40. Contre la cabane, au sud : petit crassier de $8 \mathrm{~m}^{2}$ sur $0,5 \mathrm{~m}$ d'épaisseur.

41. À $10 \mathrm{~m}$ au sud-ouest de la cabane : petit crassier de $6 \mathrm{~m} \times 4 \mathrm{~m}$ sur $0,5 \mathrm{~m}$ d'épaisseur.

42. À $70 \mathrm{~m}$ au nord-ouest de la cabane : crassier de $5 \mathrm{~m} \times 10 \mathrm{~m}$.

43. En bordure de la piste forestière, au confluent avec le vallon du Mouné : crassier en grande partie détruit par l'aménagement d'une piste forestière. Le site a fait l'objet d'un petit sondage mais le mobilier mis au jour, semblable à certains fragments de céramique du crassier $n^{\circ} 11$, ne peut être daté avec certitude.

\section{Le Versant Sud de LA Vallée du Bouchidet (fig. 10)}

44. À $50 \mathrm{~m}$ au sud de la cabane pastorale d'Artigueluz: aire de grillage de minerai d'environ $80 \mathrm{~m}^{2}$.

45. À $40 \mathrm{~m}$ au sud du $\mathrm{n}^{\circ} 44$ : aire de grillage de minerai de $5 \mathrm{~m} \mathrm{x} 10 \mathrm{~m}$.

46. Dans le vallon du Mouné, en amont de la piste forestière d'Artiguelu\%: tranchée d'exploitation de minerai de $100 \mathrm{~m}$ de longueur.
47. Sur le versant méridional du bois du Bouchidet, au lieu-dit Prat del Gaouach : crassier repéré dans une plantation de sapins inaccessible, qui n'a donc pu être mesuré.

Dans le secteur, l'étagement des activités minières et métallurgiques est particulièrement remarquable. Les sites de réduction sont localisés dans les coumes ou dans la vallée principale, bien au-dessous des aires de grillage qui sont situées à près de $1400 \mathrm{~m}$ d'altitude. Selon toute logique, on peut supposer l'existence d'une zone d'extraction un peu plus haut, près de la crête, mais celle-ci n'a pu être localisée. La distance et le dénivelé qui séparent les aires de grillage des bas fourneaux ne peuvent s'expliquer que par le souci de préserver une large bande forestière pour le charbonnage. C'est d'ailleurs le schéma adopté par les métallurgistes à une époque plus récente, comme le montrent les vestiges de nombreuses charbonnières modernes.

\section{LE COL DE Moumède (fig. 10)}

48, 49 et 50. À $15 \mathrm{~m}$ à l'ouest du col de Moumède, au milieu de la pente : trois petits crassiers accolés, d'une superficie totale inférieure à $100 \mathrm{~m}^{2}$.

51. Au sommet du col de Moumède : grande dépression sur le col qui correspond probablement à une exploitation minière. Ce secteur a simplement fait l'objet d'une exploration préliminaire. La découverte de vestiges métallurgiques dans cette zone nous conduit, pour 1999, à étendre la zone d'étude vers le nord.

\section{LE FER ET LES HOMMES DES HAUTES BARONNIES}

\section{UNE EXPLOITATION MULTISÉCULAIRE GRAVÉE DANS LE PAYSAGE}

Un simple examen des cartes de répartition des vestiges (fig. 10 à 13) et une lecture, même rapide, du catalogue mettent d'emblée en évidence la richesse des Hautes Baronnies en matière de traces d'exploitation du fer. On dénombre ainsi cinq départs d'exploitations souterraines, une vingtaine de minières, des tranchées d'extraction à ciel ouvert s'étendant sur plusieurs centaines de mètres et enfin de nombreuses traces éparses 
de sous-cavages ou de grattages. Trois aires de grillage du minerai ont été recensées, à Artigueluz et à la Crête de Sarramer, et les vestiges de réduction s'élèvent à trentetrois crassiers de scories et à trois aires d'épandage, correspondant peut-être à des crassiers arasés. S'ajoutent à ces structures proprement minières ou métallurgiques des vestiges d'habitat (Coume du Lhéris, $\mathrm{n}^{\circ}$ 6) ou d'occupation sommaire (abri sous-roche de l'Artiguette, $\mathrm{n}^{\circ} 17$ ), et les restes d'un sanctuaire (Crête de Sarramer), dont le lien avec l'activité extractive et métallurgique est fortement probable, sinon certain. Ce bilan témoigne donc éloquemment de l'intensité de l'exploitation du fer dans les Hautes Baronnies, même s'il ne peut être considéré comme exhaustif et complet. En effet, la topographie extrêmement escarpée et l'imposant couvert végétal des secteurs prospectés empêchent d'affirmer qu'aucune structure n'a échappé à la vigilance des chercheurs. Par ailleurs, la découverte, en 1998, de crassiers de scories au col de Moumède ( ${ }^{\circ s} 48$ à 50), dans la partie septentrionale des Baronnies, ouvre à la prospection de nouvelles perspectives, qui avaient été jusqu'ici négligées. En dépit des incertitudes relatives sur la fiabilité de la carte et des lacunes dans l'exploration, qui seront comblées par des prospections supplémentaires, on ne peut plus écrire, comme R. Lizop en 1931, qu' « il n'y a pas de mine de fer près d'Asque " ${ }^{54}$.

Une analyse plus détaillée de l'inventaire apporte une confirmation archéologique aux données des textes que nous évoquions en introduction : l'exploitation du fer dans les Hautes Baronnies a bien été une activité pluriséculaire, de l'Antiquité à la période contemporaine. Ainsi, les céramiques découvertes en prospection de surface dans les divers sites de la Coume du Lhéris, qu'il s'agisse de la zone d'habitat ou de sites proprement liés à l'activité minière ou métallurgique (voir $\mathrm{n}^{\text {os }} 6$ et 8 ), attestent un fonctionnement des mines et des ateliers de réduction aux périodes médiévale et moderne ${ }^{55}$. La

54. Cf. note 50, p. 38 : R. Lizop, op. cit., 1931, p. 67, note 33. Cette observation malencontreuse sert d'argument pour réfuter l'attribution à la région de l'inscription des pagani ferrarienses, que R. Lizop voulait rattacher à la localité de Ferrère dans la Barousse, distante d'une trentaine de kilomètres ( $c f$. note 49, p. 38 : R. Sablayrolles, op. cit., 2000).

55. Fn l'absence d'analyses stratigraphiques fines provenant de sondages, il est difficile de se prononcer sur l'importance relative et sur la succession précise des exploitations médiévale et moderne sur le site mentionné. Parmi le mobilier recueilli apparaît cependant de la céramique commingeoise grise, dont l'apparition n'est pas antérieure au XIVes. forge de la Gourgue d'Asque, dont la tradition orale garde le souvenir et qui a laissé quelques traces sous la forme de laitier et de scories éparses ${ }^{56}$, aurait ainsi pu être alimentée par du fer produit en amont dans l'ensemble BillexeLhéris. De même, le mobilier céramique médiéval et moderne découvert hors strati-graphie dans l'abri sousroche de l'Artiguette $\left(\mathrm{n}^{\circ} 17\right)$ témoigne peut-être d'une occupation temporaire de cet espace, liée à l'exploitation du fer. Ni l'agriculture ni le pastoralisme ne semblent en effet pouvoir justifier une quelconque installation humaine dans cette partie de la vallée, particulièrement encaissée entre des pentes très escarpées, alors que les crassiers de scories, il est vrai d'époque antique, sont distants de moins d'une centaine de mètres.

\section{LA PHASE ANTIQUE DE L'EXPLOITATION MINIÈRE ET MÉTALLURGIQUE}

Plus intéressante pour le propos initial qui était le nôtre a été la confirmation archéologique d'une phase antique de l'exploitation, que l'inscription d'Asque (fig. 18) permettait d'envisager à titre d'hypothèse de travail. Cette certitude désormais acquise est le fruit de sondages implantés sur divers crassiers dans la vallée de l'Artiguette $\left(n^{\circ} 9\right)$, la Coume des Arès $\left(n^{\circ} 18\right)$, la Crête de Sarramer ( $\left.n^{\circ} 19\right)$, la Coume des Estrets ( ${ }^{\text {os }} 28$ et 31$)$ ou la vallée du Bouchidet ( $\mathrm{n}^{\circ \mathrm{s}} 37$ et 43 , où les résultats sont cependant aléatoires). Il va de soi que la fouille du sanctuaire de Sarramer, effectuée par J.-L. Schenck et son équipe avec l'active collaboration de F. Arrouy, a constitué, dans le domaine de l'étude chronologique, un point d'ancrage essentiel. À cette notable exception près, où l'opération présentait, au-delà des objectifs chronologiques, un intérêt essentiel en matière d'archéologie religieuse pyrénéenne, l'implantation des autres sondages avait été définie soit pour vérifier par la fouille la nature des découvertes de surface, toujours rares et insuffisamment caractéristiques (crassiers de l'Artiguette et des Arès $n^{\text {}} 9$ et 18), soit en raison de la position stratégique du crassier (crassier de Sarramer n ${ }^{\circ} 19$, à égale distance du sanctuaire antique et des minières de la crête, voir fig. 17), soit pour diversifier géographiquement notre connaissance de la chronologie (sondages des Estrets et du Bouchidet, $\mathrm{n}^{\text {}} 28$,

56. X.-C. Vaussenat, $\Lambda$ bris sous-roche et vestiges de mines de fer dans la région d'Esparros, Bulletin de la sociélé Ramon, 1877, p. 115-116. 


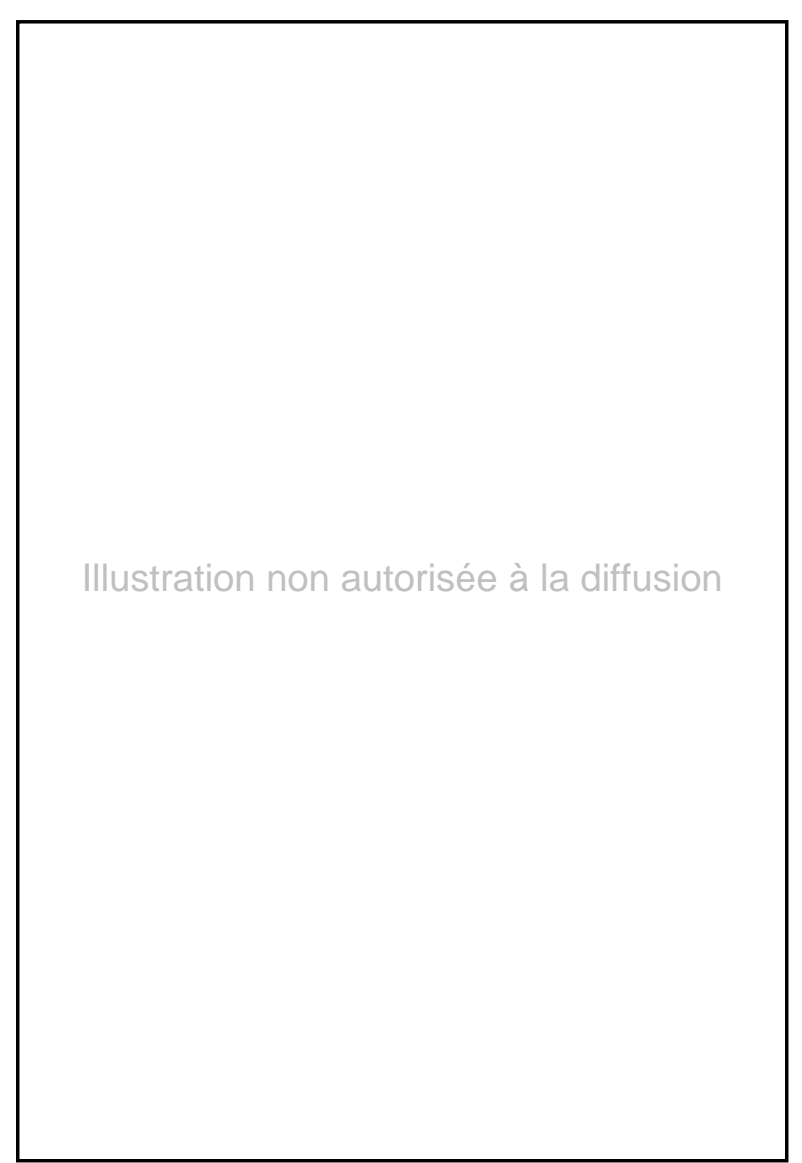

Fig. 18 - Autel votif dédié à Agcio (cliché R. Sablayrolles).

31, 37 et 43, venant compléter les données obtenues dans la partie occidentale des Baronnies). Le cas d'école le plus intéressant est sans aucun doute celui du crassier de Sarramer, situé dans un col entre le bassin du Bouchidet et celui de l'Arros, à $150 \mathrm{~m}$ au sud du sanctuaire antique et à $200 \mathrm{~m}$ au nord de la ligne des minières. La fouille a révélé, étalés sur la pente, des déchets de four de réduction et de métallurgie sur une épaisseur de 0,90 m à 1,30 m. Le mobilier céramique découvert (notamment de la céramique à paroi fine métallescente, quelques éclats de sigillée galloromaine, de forme non identifiable mais malgré tout très précieux, et des tessons de céramique commune relativement bien datés sur le site proche de Saint-Bertrand-deComminges) a permis de situer le fonctionnement de l'atelier métallurgique, modeste par sa taille et donc sans doute de courte durée, dans le courant du II ${ }^{e}$ s. de notre ère. Cette date est cohérente avec les monnaies les plus anciennes découvertes comme probables dépôts votifs dans le petit sanctuaire voisin. Par ailleurs, la situation du crassier, sur un col difficile d'accès depuis le bassin du
Bouchidet comme depuis le bassin de l'Arros, ne laisse aucun doute sur l'origine du minerai traité là : il ne pouvait s'agir que de celui que produisaient les minières de la crête, qui surplombent le col à quelque $200 \mathrm{~m}$ de distance (fig. 17). La fouille du crassier de Sarramer a donc permis d'établir et l'existence d'une coincidence chronologique entre le sanctuaire et l'activité métallurgique, et la certitude d'une phase antique d'exploitation pour les minières de Sarramer. Des remarques similaires peuvent être formulées pour le crassier des Arès $\left(\mathrm{n}^{\circ} 18\right)$, où a été découvert du mobilier du ${ }^{\mathrm{rr}} \mathrm{s}$. de notre ère et qui jouxte une tranchée d'extraction à ciel ouvert de $70 \mathrm{~m}$ de long, et pour le grand crassier de l'Artiguette $\left(n^{\circ} 9\right)$, daté par un abondant mobilier céramique des deux premiers siècles de notre ère, et qui, au confluent de la Coume d'Inher et. de la vallée de l'Artiguette (fig. 12), ne pouvait recevoir le minerai pour la réduction que des coumes situées en amont. On peut remarquer qu'aucun des sondages entrepris n'a donné de mobilier moderne et que, si les crassiers du Bouchidet ( $\mathrm{n}^{\circ} 37$ et 43 ) restent pour l'heure, dans l'attente des résultats de datation au ${ }^{14} \mathrm{C}$, mal datés, ceux des Estrets, en revanche, paraissent bien appartenir, eux aussi, à la période antique ( $\mathrm{n}^{\circ} 28$ et 31 ). Il ne fait donc aucun doute que les Hautes Baronnies ont connu une importante activité minière et métallurgique d'exploitation du fer durant l'Antiquité, dès les deux premiers siècles de notre ère au moins et peut-être jusqu'au $\mathrm{V}^{\mathrm{c}} \mathrm{s}$. si l'on accepte d'appuycr l'analyse sur la chronologie du sanctuaire et sur les quelques éléments tardifs du crassier de l'Artiguette. Cet acquis chronologique constitue un des résultats importants de l'opération et la multiplication des sondages paraît désormais suffisante pour donner à l'hypothèse valeur de certitude. Tout au plus peut-on envisager des investigations complémentaires vers la Coume de Billexe, où les repérages de surface ont fourni du mobilier nettement plus tardif (médiéval et moderne), et dans la zone septentrionale, où les découvertes de 1998 ne sont pas datées et qui constitue donc un secteur pour lequel on ne dispose, pour l'heure, d'aucune donnée précise.

\section{DONNÉES TECHNIQUES : DE PETITES EXPLOITATIONS DISPERSÉES}

Dans le domaine technique, les analyses entreprises n'ont pas encore été toutes menées à terme et, par ailleurs, la perspective initiale d'une étude chrono- 
logique, archéologique et historique induisait une démarche de recherche dans laquelle les problèmes de technique minière et métallurgique ne faisaient pas l'objet d'approche spécifique. Le sujet est donc loin d'être épuisé, toutes les potentialités du secteur en la matière n'ayant pas été explorées. Quelques observations préliminaires ne s'en dégagent pas moins, même si le compte des interrogations excède celui des certitudes.

La première évidence est la petite taille et la relative dispersion des crassiers de scories, incontestables vestiges d'ateliers de réduction du minerai. Sur les 33 crassiers repérés, 19 ont une superficie inférieure ou égale à $50 \mathrm{~m}^{2}$ (quatre d'entre eux mesurent même moins de $10 \mathrm{~m}^{2}$ ), 4 ont une superficie comprise entre 50 et $200 \mathrm{~m}^{2}, 5$ seulement mesurent plus de $200 \mathrm{~m}^{2}$, les « grands » crassiers de l'Artiguette ( $\mathrm{n}^{\mathrm{os}} 9$ et 11) ayant respectivement pour surface 600 et $450 \mathrm{~m}^{2}$ (le premier comportant une épaisseur de déchets de métallurgie supérieure à $3 \mathrm{~m}){ }^{57}$. Ces chiffres n'ont rien de comparable, on le voit, avec les grands crassiers de la Montagne Noire, qui mesuraient plusieurs hectares de superficie et où les scories s'entassaient sur plus de $10 \mathrm{~m}$ de hauteur ( $c$. Decombeix et al., supra, p. 23-36). Le contraste entre le volume des scories et la taille de certaines exploitations minières est même saisissant, comme dans la Coume des Arès où le petit crassier du $\mathrm{I}^{\mathrm{er}} \mathrm{s}$. de notre ère $\left(150 \mathrm{~m}^{2}\right.$ de surface et une épaisseur de scories ne dépassant pas $1 \mathrm{~m}$ ), totalement isolé, jouxte une tranchée d'exploitation du minerai longue de $70 \mathrm{~m}$, large de 4 à $10 \mathrm{~m}$ et profonde de 5 à $10 \mathrm{~m}^{58}$. Ces observations, que l'on pourrait réitérer autour de la Crête de Sarramer par exemple, témoignent à l'évidence que l'ensemble du minerai extrait n'a pas été réduit en totalité dans les coumes attenantes. Cela laisse ouverte, et probable, l'hypothèse d'une réexploitation à diverses époques des mêmes gisements miniers avec des destinations différentes pour la réduction. Pour la phase antique elle-même, bien documentée, on l'a vu, puisque tous les crassiers sondés appartiennent pratiquement à cette période, on ne peut écarter totalement l'hypothèse d'ateliers de réduction plus éloignés, vers les

57. La superficie de 5 crassiers n'a pu être mesurée, soit parce qu'ils étaient partiellement détruits par les pistes forestières (crassiers du Bouchidet ou de Oueil Luzent), soit parce qu'ils étaient inaccessibles, enfermés dans des espaces clôturés par l'Office national des forêts.

58. Il s'agit là de la profondeur actuelle de la tranchée, dont le fond est recouvert d'un épais tapis végélal. Ia profondeur de l'exploilation était évidemment plus importante.
Basses Baronnies par exemple, bien qu'aucun crassier n'ait été jusqu'ici signalé dans ces secteurs du piémont.

La relative dispersion des crassiers n'exclut pas cependant un schéma rationnel d'exploitation. L'analyse de la carte de répartition, au contraire, met en évidence des constantes qui tiennent compte de la réalité du relief et de la géologie. On constate ainsi que la plupart des vestiges de réduction se rencontrent au débouché de petites coumes dans les vallons principaux que constituent les vallées de l'Artiguette, du Bouchidet, de l'Arros et du Lhéris. C'est particulièrement net pour les crassiers de l'Artiguette, où les trois ensembles les plus imposants sont concentrés au débouché de la Coume d'Inher dans la vallée de l'Artiguette (fig. 12, $\mathrm{n}^{\text {os }} 9-11$ ), et où les petits crassiers repérés en aval de ce groupe occupent le confluent entre la vallée principale et de petits vallons à l'accès particulièrement difficile (fig. 12, $\mathrm{n}^{\mathrm{os}}$ 12-15). Ce mode de répartition topographique se répète pour les crassiers des Estrets et du Bouchidet et, de façon encore plus nette, pour les crassiers de la Coume du Lhéris au pied de la montagne de Billexe. La présence de zones de grattage ou de sous-cavage dans les parties hautes des petites coumes, voire d'exploitations souterraines comme dans le cas de la Coume d'Inher et de celle du Lhéris, offre l'explication du phénomène : les ateliers de réduction étaient installés au plus près des lieux d'extraction, dès qu'étaient réunis un espace relativement moins escarpé et la proximité de l'eau, toutes conditions qu'offraient les confluents des coumes et des vallées principales. Ce schéma directeur, dont les exemples sont nombreux, n'était cependant pas unique : le crassier de la Crête de Sarramer $\left(n^{\circ} 19\right)$ et celui de la Coume des Arès $\left(n^{\circ} 18\right)$, tous deux d'époque antique, occupent certes une position très proche des lieux d'extraction, mais ils sont situés, le premier sur un col à $1205 \mathrm{~m}$ d'altitude, le second sur une crête à $1025 \mathrm{~m}$ d'altitude, très loin de toute confluence et de tout point d'eau. La proximité immédiate du minerai paraît, dans ces deux cas, avoir constitué l'élément déterminant du choix d'implantation de l'atelier.

Dans le domaine proprement technique, nous l'avons dit, ni les investigations ni les analyses sur la base de données existante ne sont terminées. Ce sont donc plus des perspectives de recherche que des conclusions que l'on peut définir. La coexistence d'exploitations à ciel ouvert, sous forme de minières ou de tranchées comme à la 
Crête de Sarramer où se rencontrent les deux types de structure, et d'exploitations souterraines, dont témoignent plusieurs départs de galeries, est une certitude. Il est d'ailleurs possible que les plus grandes minières de la Crête de Sarramer constituent la partie supérieure de dépilages en partie souterrains, désormais obstrués par les déblais accumulés en raison de l'érosion naturelle ${ }^{59}$. On ne peut affirmer, cependant, que les deux formes d'extraction soient contemporaines, les seules structures datées étant, de façon indirecte par la proche présence des crassiers, les minières de Sarramer (ou au moins certaines d'entre elles) et la tranchée de la Coume des Arès, qui appartiennent au moins partiellement à l'époque antique. Peut-on espérer en apprendre plus? Dater plus précisément les minières ou les exploitations souterraines supposerait un travail préliminaire particulièrement lourd de désobstruction pour un résultat a priori relativement aléatoire. Il faudrait en effet atteindre les niveaux contemporains de l'exploitation, qui peuvent être recouverts de plusieurs mètres de déblais dans le cas des minières et obstrués par des effondrements dans le cas de travaux souterrains. Cela demanderait l'utilisation d'engins lourds, difficiles à amener à $1300 \mathrm{~m}$ d'altitude par des pistes souvent transformées en fondric̀res. Par aillcurs, ces nivcaux d'cxploitation sont susceptibles d'avoir subi de considérables altérations lors de réexploitations successives et, en tout état de cause, l'expérience montre qu'ils contiennent rarement des indicateurs chronologiques fiables et précis, même quand ils sont intacts. L'enjeu paraît donc insuffisant, la disproportion entre la taille des moyens à mettre en œuvre et le résultat raisonnablement envisageable étant trop considérable.

Dans le domaine de la métallurgie, la découverte de nombreuses tuyères scorifiées dans les crassiers de la Crête de Sarramer et de la Coume des Arès ne manque pas de poser problème. En effet, par leur forme (partie inférieure aplatie, constituant une surface de pose) comme par leur structure (gangue de scories entourant une paroi sablo-argileuse durcie par la chaleur), elles ne paraissent pas pouvoir être rattachées au fonctionnement des bas fourneaux de réduction. Faut-il songer à des tuyères de forge ou de foyer d'affinage, qui auraient été utilisées pour le cinglage du massiot dès sa sortie du

59. Voir sur cc phćnomc̀nc lcs observations faites par B. Cauuet dans les aurières du Limousin (Cauuet, 1992, notamment p. 8 et fig. 3). four? C'est l'hypothèse la plus vraisemblable, que devrait confirmer l'analyse actuellement en cours de microparticules de métal prélevées à la surface des tuyères et qui pourraient constituer des battitures de cinglage. Une plaque scoriacée, récupérée dans le crassier des Arès, s'est révélée à l'analyse être un fond de foyer d'épuration ou de forge, preuve que l'opération de cinglage ou d'affinage s'effectuait bien à même le lieu de réduction du minerai ${ }^{60}$.

La découverte d'une aire de grillage en parfait état de conservation à la Crête de Sarramer $\left(n^{\circ} 21\right)$ offrait elle aussi des perspectives d'étude des techniques. Le minerai traité, dont une couche était encore in situ, est de l'hématite, dont le grillage a considérablement augmenté la proportion d'oxyde de fer $\left(\mathrm{Fe}_{2} \mathrm{O}_{3}: 82,5 \%\right)^{6 !}$. Le bon état de la structure bâtie, sa situation stratigraphique, pratiquement à même le sol actuel, la conservation parfaite des charbons de bois laissent cependant penser qu'il s'agit d'un élément moderne voire contemporain, peut-être une aire artisanale où était chauffé du minerai plus ancien récupéré dans des haldes voisines, visiblement bouleversées par une réexploitation. En l'absence de tout fossile directeur, il faut attendre les résultats d'une datation au ${ }^{14} \mathrm{C}$ pour se prononcer sur la chronologie et la destination exacte de cette aire de grillage.

Une dernière question technique d'importance est l'impact qu'a pu avoir sur la forêt avoisinante l'exploitation métallurgique, l'utilisation, et donc la fabrication, in situ, du charbon de bois étant le corollaire inévitable de l'installation de fourneaux de réduction. Une cartographie des charbonnières, qui n'est pas entièrement terminée, a été effectuée par l'équipe de J.-P. Métailié (GEODE, UMR 966 du CNRS, Toulouse). Elle semble mettre en évidence la présence de ce type de structures aux alentours de la zone métallurgique proprement dite, où, en revanche, ces charbonnières sont plus rares. Elles sont particulièrement nombreuses sur les

60. Ces identifications, faites au laboratoire de Minéralogic de l'université Paul-Sabatier Toulouse III par $\mathbf{F}$. Tollon, directeur de recherche au CNRS, ont été rendues possibles par l'analyse de la structure de la plaque, qui portait la trace de plusieurs opérations successives, par la richesse du matériau en magnétite et par la présence, sur la paroi intérieure, de petites billes millimétriques, les battitures, résidus du cinglage.

61. Les analyses des échantillons ont été effectuées au laboratoire de Minéralogie de l'université Paul-Sabatier Toulouse III par F. Tollon. 
pentes septentrionales de la vallée du Bouchidet, entre la Crête d'Artigueluz et le fond de la vallée, mais il s'agit là de structures contemporaines, comme en témoignent les nombreux vestiges de cuvelage en fer épars sur le site. D'autres charbonnières, cependant, situées vers le massif du Bassia, paraissent plus anciennes, ancienneté que devraient éventuellement confirmer des datations au ${ }^{14} \mathrm{C}$. Dans ce cas de figure une étude plus poussée serait justifiée, avec sondages stratigraphiques d'une ou de plusieurs charbonnières et analyses anthracologiques des résidus charbonneux. Une étude palynologique avait été également envisagée en collaboration avec D. Galop, chercheur au CNRS, l'objectif étant de mesurer par l'analyse quantitative et qualitative des pollens l'éventuelle déforestation liée à l'implantation de l'activité métallurgique, de l'Antiquité à la période moderne. Malheureusement, aucune tourbière adéquate pour ce type d'investigation n'a pu être trouvée dans un cadre voisin des Hautes Baronnies, le seul placage tourbeux du secteur, au col de Couradabat, étant piétiné et bouleversé par les animaux à l'estive. Les résultats obtenus sur le plateau de Lannemezan, distant de plus d'une dizaine de kilomètres, et dans la région de Montréjeau, à plus de vingt kilomètres, ne sauraient, malgré leur intérêt pour la période antique, être rattachés au complexe métallurgique des Hautes Baronnies.

\section{PAGUS FERRARIENSIS}

Le bilan de la prospection et des sondages ne laisse, nous l'avons vu, aucun doute sur l'origine de l'inscription d'Asque: les pagani ferrarienses, qui honoraient sur un autel votif la divinité locale Ageio, étaient bien les habitants d'un pagus qui tirait son nom de l'exploitation toute proche du fer des Hautes Baronnies. La divinité, attestée par ailleurs sur cinq inscriptions de la région, était également présente dans le petit sanctuaire d'altitude situé à proximité immédiate des minières et du crassier de la Crête de Sarramer ${ }^{62}$. Le rapprochement de ces diverses données

62. Inscriptions au dieu Ageio: CIL XIII, 180 (Montégut, en basse vallée de Neste, Ilautes-Pyrénées), 221 (Rebouc, près de Hèches en vallée d'Aure, Hautes-Pyrénées) et 383, 385, 386 (trouvées dans la région d'Asque, au débouché de l'Arros dans les Basses Baronnies). Un des petits autels découverts dans le sanctuaire de Sarramer porte le nom de la divinité, très ćrodé mais encore lisible. suggère quelques réflexions en matière d'histoire culturelle et religieuse, mais ouvre aussi des perspectives sur un domaine qui, le plus souvent, échappe à l'analyse : la condition des exploitants dans le cadre de l'activité minière et métallurgique.

Sans revenir en détail sur des thèmes qui ont été largement traités par ailleurs ${ }^{63}$, il faut, pour les besoins de la démonstration, rappeler brièvement que ces inscriptions votives pyrénéennes révèlent une série de superpositions, d'amalgames, de transformations dans le tissu économique, social et culturel d'une communauté indigène qui rencontre la culture romaine au gré de mutations politiques, administratives mais aussi et surtout économiques. Il est ainsi clair que l'exploitation des ressources naturelles (bois, pierre, minerai), élément fondamental d'une économie montagnarde traditionnelle, accentue, par le biais de l'échange, le processus d'intégration de la communauté autochtone à la communauté impériale romaine. L'exemple de la divinité Ageio est, en ce sens, parfaitement parallèle à celui des dieuxarbres ${ }^{64}$ ou de la divinité Erriape des marbriers de SaintBéat ${ }^{65}$. Divinité protectrice de la communauté originelle, et limitée donc dans son influence à l'espace géographique restreint qui constitue le bassin de vie de cette communauté, le dieu devient, peu à peu et avec le temps, la divinité protectrice de la structure administrative (le pagus), qui recouvre plus ou moins le territoire originel, et la divinité protectrice de l'activité économique qui constitue la raison d'être principale du pagus, carrières de marbre dans le cas d'Erriape à Saint-Béat, mines et métallurgie du fer dans le cas d'Ageio dans les Hautes Baronnies. L'expression du rituel et la culture des fidèles

63. R. Sablayrolles, J.-L. Schenck, Collections du musée archëologique départemental de Saint-Bertrand-de-Comminges -I- Ies aulels votifs, Saint-Bertrandde-Comminges, 1988; R, Sablayrolles, J.-I. Schenck, Autels iotifs, Catalogue de l'exposition du musée archéologique départemental de Saint-Bertrand-de-Comminges (1990-1991), Saint-Bertrand-deComminges, 1990 ; R. Sablayrolles, Les dieux des bûcherons dans les Pyrénées à l'époque de la domination romaine, in : Protoindustries et histoire des forêts, Actes du colloque tenu à la Maison de la Forêt (Loubières, Ariège) les 10-13 oct. 1990, Toulouse, 1992, p. 15-26 ; J.-M. Fabre, R. Sablayrolles, I ee dicu Frriape et les isotopes stables : les carric̀res des Pyrénćcs, cntre terrain et laboratoire, Entretiens d'archéologie et d'histoire 2, Saint-Bertrand-de-Comminges, in: Les marbres blancs des Pyrénées: approches scientifiques el historiques, Saint-Bertrand-deComminges, 1995, p. 130-168; cf. note 49, p. 38 : R. Sablayrolles, op. cit., 2000 et à parâtrte.

64. Cf. note 63: R. Sablayrolles, op. cit., 1992.

65. Cf. note 63: J.-M. Fabre, R. Sablayrolles, op. cil., 1995. 
(onomastique notamment) se romanisent d'autant plus que l'activité économique et l'échange se développent ${ }^{66}$. Parallèlement, la personnalité de la divinité s'assimile, en se déformant, à celle de divinités du panthéon romain, sans que jamais se perde complètement son caractère indigène. Dieu de la communauté des Hautes Baronnies, Ageio devint ainsi le protecteur du pagus romain en même temps que celui de la principale activité de ce pagus: l'exploitation du fer qui lui avait valu son épithète de ferrariensis. En témoignent l'inscription d'Asque et le sanctuaire d'altitude de Sarramer, dont le lien avec l'activité métallurgique est indéniable, tant en raison de la proximité des diverses structures qu'en raison de la nature de certains objets déconverts dans le sanctuaire.

Les analyses du corpus des fidèles de ces divinités pyrénéennes montrent qu'il s'agissait majoritairement, pour ne pas dire exclusivement, d'autochtones romanisés che $\iota$ qui subsistaient de fortes traditions, notamment onomastiques, empruntées à une langue non indoeuropéenne, parlée antérieurement au latin et au celte, et que, faute de mieux, on dénomme aquitaine ${ }^{67}$. Ie recours à l'onomastique latine et l'usage chez certains fidèles des tria nomina témoignent de la romanisation plus grande d'une frange minoritaire de cette population, dont certains membres avaient le statut de citoyen. Globalement, les dieux liés à une activité économique ouvrant sur l'échange (Erriape et exploitation du marbre, dieux-arbres et exploitation du bois) possèdent des groupes de fidèles plus romanisés que les autres, et le statut de citoyen s'y rencontre plus fréquemment ${ }^{68}$. G. Fabre a même montré que c'est vraisemblablement l'élite économique de cette société, quelques grandes familles liées à diverses formes d'exploitation de la richesse montagnarde, qui avait facilité, par son intégration à la citoyenneté et son acculturation, la romanisation du panthéon local et l'adoption de rites et de formes de dévotion latins pour des dieux qui conservaient leur nom indigène même quand leur personnalité se modifiait au gré des réalités nouvelles ${ }^{69}$. Ageio appar-

66. Cf. note 63 : R. Sablayrolles, op. cit., 1992.

67. Cf. note 63 : R. Sablayrolles, J.-L. Schenck, op. cit., 1988 et 1990.

68. Cf. note 63 : R. Sablayrolles, op. cit., 1992 et à paraître.

69. J.-M. Fabre, La dimension sociale de l'interpretatio des divinités locales en Aquitaine méridionale durant l'Empire romain, in: Divinidades indigenas e interpretatio romana, II Coloquio internacional de Epigrafia, Sintra (Portugal), 16-18 mars 1995, à paraître. tient bien à cette catégorie : en dehors des pagani ferrarienses, l'ont honoré d'une inscription un I abusius dont le gentilice, utilisé comme nom unique, constitue un hapax, un [P]aulini/[us T]aurini (filius), dont les surnoms comme la dénomination pérégrine révèlent un pyrénéen libre latinisé, et deux citoyens: Antonius Vindemialis et Metel[lini]a Qu[inta] ${ }^{70}$.

Ces observations offrent matière à réfléchir sur la condition des métallurgistes antiques des Hautes Baronnies et la gestion des exploitations. Si on veut bien admettre le lien entre le sanctuaire de Sarramer et l'activité minière et métallurgique toute proche, les fidèles qui rendaient là de modestes hommages à Ageio étaient probablement, comme tous les dévots des dieux pyrénéens, en majorité des pérégrins libres d'origine locale. Une partie de la main-d'œuvre associée à l'entreprise minière et/ou métallurgique était donc constituée d'une population locale libre, de condition sociale modeste, peutêtre peu latinisée, mais qui avait accès aux produits des grands circuits d'échange de l'Empire romain, notamment monnaies et céramique sigillée, et qui utilisait le support des autels votifs pour ses dévotions, comme en témoignent les découvertes du mobilier lapidaire et numismatique du sanctuaire ou divers fragments de céramique jetés dans le crassier de scories tout proche ${ }^{71}$. Antonius Vindemialis, par ailleurs, dont l'inscription à Ageio fut découverte à Montégut en basse vallée de la Neste, est connu par une autre inscription

70. Labusius : CIL XIII, 386 (= Sacaze, 1892, n 464); seul exemple proche, un M. Laburius Gratus à Narbonne (CIIL XII, 5204). Les surnoms Paulinus íqui a pris sur l'inscription la forme d'un gentilice: Paulinius) et Taurinus font partie des surnoms latins les plus répandus parmi le milieu pyrénćen ( $c f$. note $63:$ R. Sablayrolles, J.-L. Schenck, op. cit., 1988, p. 43 , note 2 et p. 45, note 2). Antonius Vindemialis ( $C I L$ XIII, $180=$ Sacaze, $1892, \mathrm{n}^{\circ} 452$ ) porte un surnom peu répandu (8 exemples seulement répertoriés par I. Kajanto, dont 5 en Afrique, cf. Kajanto, The Latin Cognomina, Commentationes Humanarum Lillerarum, Societas Scientiarum Fennica, XXXVI, Hclsinki, 1965, p. 218), ce qui permet de l'identifier au dédicant d'une inscription à Jupiter trouvée en vallée de Barousse, près de Saint-Bertrand-de-Comminges (CIL XIII, 308 = Sacaze, 1892, n 391). La lecture Metillinia Quinta, suggérée par le dessin de J. Sacaze (1892, $\left.\mathrm{n}^{\circ} 465\right)$, est préférable à la lecture du Corpus : Metellini]/.ia ov (CIL XIII, 383).

71. La nature des données ne permettra jamais d'affirmer que le sanctuaire était celui des mineurs ou des métallurgistes, mais l'association des fidèles du sanctuaire à l'activité voisine, quelle que soit sa forme, paraît indéniable. L'analyse détaillée de la structure du sanctuaire et du mobilier scra donnéc dans la publication que prépare J.L. Schenck. Nous le remercions bien vivement de nous avoir autorisés à utiliser ici certaines des informations dont il nous a fait part. 
votive découverte en vallée de Barousse, près de SaintBertrand-de-Comminges, et dédiée à Jupiter Très Bon et Très Grand (CIL XIII, 308). L'identité des deux dédicants étant certaine (voir note 70), G. Fabre voit dans le personnage un de ces aristocrates locaux possédant des intérêts dans diverses vallées des Pyrénées centrales, aristocrates dont l'intégration à la citoyenneté et l'adaptation à la culture romaine servaient de modèle aux populations pyrénéennes, facilitant l'adoption de rites et de cultes romains et favorisant le phénomène d'interpretatio des divinités ${ }^{72}$. Peut-on aller plus loin et attribuer à cet Antonius Vindemialis, fidèle d'Ageio comme de Jupiter, des intérêts dans le fonctionnement des mines de fer et des ateliers de réduction ? À l'appui de cette hypothèse, on pourrait souligner que l'inscription de Barousse, découverte dans le village d'Ilheu, est proche de Ferrère, localité dont le toponyme, attesté dès le $\mathrm{X}^{\mathrm{e}} \mathrm{s}$., évoque l'exploitation du fer. L'argument paraît toutefois insuffisant pour faire en toute certitude d'Antonius Vindemialis une sorte de capitaine d'industrie local, qui aurait investi dans la production du fer à Ferrère en Barousse et dans les Hautes Baronnies, patrie d'Ageio. Le lien topographique entre l'inscription d'tlheu et le village de Ferrère est en effet mal assuré (les deux localités sont distantes d'une dizaine de kilomètres) et l'antiquité de l'exploitation du fer à Ferrère reste encore archéologiquement à démontrer ${ }^{73}$. Il n'est pas inutile de remarquer cependant que la petite taille et la dispersion des crassiers, que nous avons soulignées, contrastent avec les grands ensembles de la Montagne Noire, dont on

72. Cf. note $69:$ J-M. Fabre, op. cit., à paraître.

73. Des prospections menées en 1987 par G. Pradalić et R. Sablayrolles n'ont permis de retrouver que deux crassiers de scories d'époque indéterminée, dont un situé sous l'église du village. Le filon de fer de la vallée de la Serviassa, en amont de Ferrère, a été réexploité en galerie dans les années 1910-1920. Seule la clatation du toponyme, attesté dès le $\mathrm{X}^{\mathrm{c}} \mathrm{s}$., constitue un terminus ante quem pour l'exploitation du fer. attribue la propriété ou l'affermage aux grandes sociétés de publicains de la fin de la République (Domergue dir., 1993, p. 409). Faut-il penser que les exploitations des Hautes Baronnies appartenaient à un mode de gestion différent, où la cité voire l'initiative privée tenaient une plus grande place? Ce n'est pas impensable: les mines de fer échappèrent plus longtemps que les autres au contrôle étatique et l'hypothèse d'exploitations de taille modeste relevant d'une gestion municipale ou privée n'est pas à exclure en Gaule au moins jusqu'au début du III' s. (Sablayrolles, 1989a).

Si ces dernières hypothèses sont fragiles et constituent au mieux des perspectives de recherche, la participation d'une main-d'œuvre locale libre à l'exploitation paraît, par contre, indéniable. Elle pose une dernière question : cette participation est-elle le fruit de la romanisation, qui aurait ouvert les mines de fer des Hautes Baronnies comme elle avait ouvert les carrières de marbre de SaintBéat, ou la conséquence d'une tradition plus ancienne, que la présence romaine et les changements économiques qu'elle entraînait auraient développée et transformée? En d'autres termes, les mots pagus ferrariensis sont-ils la traduction littérale d'une appellation antérieure ou la création de l'administration romaine et des mutations économiques qu'elle reflétait? Aucune réponse archéologique n'existe pour l'heure à la question, puisqu'aucune trace d'exploitation préromaine du fer n'a été jusqu'ici mise au jour, et on ne saurait se réfugier derrière les descriptions de César, vantant les qualités de sapeur des Aquitains (César, La Guerre des Gaules, 3, 21, 3), pour faire des Pyrénéens des Hautes Baronnies des mineurs préromains : ce serait retomber dans les travers que nous dénoncions en commençant notre propos ${ }^{74}$.

74. Le texte en question concerne, au demeurant, les Sotiates, Aquitains des plateaux de Gascogne, et fait allusion à des mines de cuivre (aerariae) et à des carric̀res (sectur(te). 